Periodontal diseases are localized chronic inflammatory conditions of the gingival and underlying bone and connective tissue. Platelet-activating factor (PAF), a potent inflammatory phospholipid mediator that has been previously detected in elevated levels in inflamed gingival tissues, in gingival crevicular fluid and in saliva, is implicated in periodontal disease. Our results from previous studies showed that the biologically active phospholipid detected in gingival crevicular fluid is a hydroxyl-PAF analogue. In this study, hydroxyl-PAF analogue was detected for the first time in human blood derived from patients with chronic periodontitis as well as from periodontally healthy volunteers. The hydroxyl-PAF analogue was purified by high-performance liquid chromatography, detected by biological assays and identified by electrospray analysis. In addition, the quantitative determination of PAF and hydroxyl-PAF analogue (expressed as PAF-like activity) showed a statistically significant increase in the ratio of hydroxyl-PAF analogue levels to PAF levels in periodontal patients, suggesting that this bioactive lipid may play a role in oral inflammation.

Key words: Platelet-activating factor, Gingival crevicular fluid, Periodontal disease, Electrospray mass spectrometry, Phospholipids

\section{Hydroxyl-platelet-activating factor exists in blood of healthy volunteers and periodontal patients}

\author{
Smaragdi Antonopoulou ${ }^{1, C A}$, Alexandros Tsoupras ${ }^{2}$ \\ George Baltas ${ }^{3}$, Helen Kotsifaki ${ }^{4}$, \\ Zacharias Mantzavinos ${ }^{3}$ and \\ Constantinos A. Demopoulos 2
}

${ }^{1}$ Department of Science of Dietetics - Nutrition, Harokopio University, 70 El. Venizelou str., 17671 Athens, Greece; ${ }^{2}$ Faculty of Chemistry, National and Kapodistrian University of Athens,

Panepistimioupolis, Athens, Greece; ${ }^{3}$ Department of Periodontology, National and Kapodistrian University of Athens, Greece; ${ }^{4}$ Department of Physiology, National and Kapodistrian University of Athens, Greece

\footnotetext{
${ }^{\mathrm{CA}}$ Corresponding Author

Tel: +32 109549305

Fax: + 32109577050

E-mail: antonop@hua.gr
}

\section{Introduction}

Periodontal disease is a chronic disorder that occurs in susceptible individuals and is initiated when microorganisms of dental plaque (i.e. Porphyromonas gingivalis, Actinobacillus actinomycetemcomitans, Treponema dentocola) colonize the gingival sulcus, and thereafter the periodontal pocket. The inflammatory response by the host results in destruction of periodontal tissues and alveolar bone loss. ${ }^{1}$

Several reviews have summarized the role of activated inflammatory cells (e.g. polymorphonuclears, macrophages and lymphocytes) when challenged by bacterial lipopolysaccharide, as a key regulator of periodontal disease expression. These cells produce and secrete proinflammatory mediators such as interleukin- $1 \beta$ and tumour necrosis factor- $\gamma$ that induce and enhance the production of prostaglandin $\mathrm{E}_{2}$ and matrix metallo-proteinases. These molecules mediate destruction of the extracellular matrix of periodontal tissues and resorption of the alveolar bone. ${ }^{2,3}$

Platelet-activating factor (PAF), a potent inflammatory phospholipid mediator that has been previously detected in elevated levels in inflamed gingival tissues and gingival crevicular fluid (GCF), is implicated in periodontal disease. ${ }^{4,5}$

Our results from previous studies obtained from chemical and enzymatic treatments, and biological assays as well as from electrospray analysis showed that the biologically active phospholipid detected in GCF is not a typical PAF molecule (1-O-alkyl-2acetyl-sn-glycero-3-phosphocholine), ${ }^{6}$ but a hydroxyl-PAF analogue. ${ }^{7}$

Studies have also revealed that PAF is actually a family of structurally related acetylated phospholipids, which possesses potent inflammatory activities. ${ }^{8}$ It has also been reported that salivary PAF levels are increased in periodontitis, most probably originated from the crevicular space and derived from inflammatory cells within the gingival and/or periodontal tissues. $^{9,10}$

In addition, recent cross-sectional and prospective epidemiological studies have demonstrated an association between periodontal disease and atherosclerosis and human coronary heart disease. ${ }^{11-13}$ Periodontitis and cardiovascular disease share risk factors including tobacco smoking, male gender, 
race, age, education, poverty index, diabetes, systemic hypertension and hypercholesterolaemia. These commonalities suggest that periodontal disease and heart disease may also have many pathogenetic mechanisms in common. It is possible that infection and chronic inflammatory condition such as periodontitis may contribute to the pathogenesis of atherosclerosis. The chronicity of periodontal disease provides a rich source of bacterial and host response products for a long period of time. Two main processes may provide an etiological link between these two diseases: the bacterial lipopolysaccharide and the monocyte-related responses. ${ }^{2,14}$

It should be noted that PAF and the other unique acetylated phospholipids are produced by a variety of cells that participate in the development of inflammatory reaction such as monocytes/macrophages, polymorphonuclear neutrophils and platelets. ${ }^{8,15}$ PAF, and mostly PAF-like oxidized phospholipids, are implicated in atherosclerosis and cardiovascular diseases. ${ }^{16-20}$ These findings suggest that inflammatory mediators such as the hydroxylPAF analogue, which was identified in GCF, may also be present in blood.

The purpose of this study was to detect and identify the hydroxyl-PAF analogue in human blood and to have a first approach on evaluation of the relationship between its levels and the periodontal inflammation.

\section{Methods}

\section{Human subjects}

In this study, five patients with chronic adult periodontitis and five periodontally healthy volunteers participated. Both groups consisted of males, aged 32-41 years; none of them had received any periodontal treatment or antibiotic therapy during the past 6 months. The characterization of periodontitis was based on the measurements of clinical parameters, plaque index, bleeding on probing and probing depth. ${ }^{5}$

\section{Solvents and reagents}

All reagents and chemicals were analytical grade supplied by Merck (Darmstadt, Germany). Highperformance liquid chromatography (HPLC) solvents were from Rathburn (Walkerburn, UK) Lipid standards, bovine serum albumin (BSA) and thrombin were obtained from Sigma (St Louis, MO, USA). Semisynthetic PAF (80\% C-16PAF and 20\% C-18PAF) was synthesized in our laboratory as previously described. $^{6}$ PAF-acetylhydrolase was from human serum, purified according to the method of Stafforini et al. ${ }^{21}$ Silicic acid, 35-70 mesh, ASTM 7733 (Merck), was used for column chromatography.

\section{Instrumentation}

The separation of lipids was performed at room temperature on a HP HPLC Series 1100 liquid chromatography model (Hewlett Packard, Waldbronn, German) equipped with a $100 \mu$ loop Rheodyne (7725 i) loop valve injector, a degasser G1322A, a quat gradient pump G1311A and a HP UV spectrophotometer G1314A as a detection system. The spectrophotometer was connected to a HewlettPackard model HP-3395 integrator-plotter. PAF-induced aggregation was measured in a Chrono-Log (Havertown, PA, USA) aggregometer (model 400-VS) coupled to a Chrono-Log recorder. The electrospray ionization (ESI) mass spectrometry experiments were performed on an API 100 Perkin Elmer SCIEX single quadrupole mass spectrometer. Samples were dissolved in a small volume of HPLC-grade methanol/ water $(70: 30, \mathrm{v} / \mathrm{v}), 0.01 \mathrm{M}$ in ammonium acetate. Electrospray samples are typically introduced into the mass analyser at a rate of $4.0 \mu \mathrm{l} / \mathrm{min}$. The positive and negative ions, generated by charged droplet evaporation, enter the analyser through an interface plate and a $100 \mathrm{~mm}$ orifice, while the de-clustering potential is maintained between 60 and $100 \mathrm{~V}$ to control the collisional energy of the ions entering the mass analyser. The emitter voltage is typically maintained at $4000 \mathrm{~V}$. The electrospray ionization tandem mass spectrometry (MS/MS) experiments were performed on a Finnigan LCQ quadrupole ion trap mass spectrometer. Electrospray samples were typically introduced into the mass analyser at a rate of 4.0 $\mu \mathrm{l} / \mathrm{min}$. The positive and negative ions, generated by charged droplet evaporation, entered the analyser through a heated capillary plate. The emitter voltage was typically maintained at $4000 \mathrm{~V}$.

\section{PAF and hydroxyl-PAF analogue isolation and purification}

The isolation and purification of PAF and hydroxylPAF analogue was performed according to the method of Demopoulos et al. ${ }^{22}$ Briefly, $20 \mathrm{ml}$ of blood were collected from each human subject and, immediately after collection, were poured into $80 \mathrm{ml}$ of absolute ethanol and centrifuged at $1000 \times g$ for $20 \mathrm{~min}$. The supernatant and the pellet were extracted according to the Bligh-Dyer method, ${ }^{23}$ and the phased chloroforms were mixed. The extracts were purified on silicic acid column chromatography that is eluted with $45 \mathrm{ml}$ of methanol/water $(1: 1.5, \mathrm{v} / \mathrm{v})$, followed by $50 \mathrm{ml}$ of methanol/water $(2: 1, \mathrm{v} / \mathrm{v})$. The initial $45 \mathrm{ml}$ (containing the bulk of proteinaceous and other non-lipid impurities) were discarded while the PAF containing eluents 
were further purified by HPLC. A normal phase column, Sphereclone $5 \mathrm{u} \mathrm{NH} \mathrm{NH}_{2} 250 \times 4.6 \mathrm{~mm} \mathrm{I.D.}$, from Phenomenex (Hurdsfield, UK), was used. ${ }^{24}$ The solvent system consisted of an isocratic elution with 100\% solvent A (acetonitrile/methanol, 70:30, v/v) for 35 min followed by a linear gradient to $100 \%$ solvent $\mathrm{B}$ (methanol) in $5 \mathrm{~min}$, a hold for $5 \mathrm{~min}$ in 100\% solvent B followed by a linear gradient to $100 \%$ solvent C (water) in $5 \mathrm{~min}$, and finally a hold in 100\% solvent $\mathrm{C}$ for $10 \mathrm{~min}$. The eluted substances were detected using ultraviolet detection at $208 \mathrm{~nm}$.

\section{Biological assays}

Semi-synthetic PAF and the examined samples were dissolved in $2.5 \mathrm{mg} \mathrm{BSA} / \mathrm{ml}$ of saline. Thrombin was dissolved in saline. The PAF and the hydroxyl-PAF activity were estimated by measuring the biological activity towards washed rabbit platelets, comparing the PAF-induced aggregation with known concentra- tions of semi-synthetic PAF. Experiments with specific inhibitor BN 52021, 0.1 mM (0.3\% dimethylsulphoxide in water), were also performed. This inhibitor was added to the aggregometer cuvette containing washed rabbit platelets $1 \mathrm{~min}$ prior to the addition of the examined sample. This experiment was carried out according to Lazanas et al. ${ }^{25}$ In desensitization and cross-desensitization experiments, platelets were desensitized by the addition of the test lipid to the platelet suspension at a concentration that caused reversible aggregation. Second stimulation with PAF or thrombin was performed immediately after complete disaggregation.

\section{Treatment with acetylhydrolase}

The effect of PAF acetylhydrolase of human serum, an enzyme specific to short or intermediate length $s n$ 2 chains, on the ability of fractions to induce platelet aggregation was examined. Briefly, Tris buffer (50

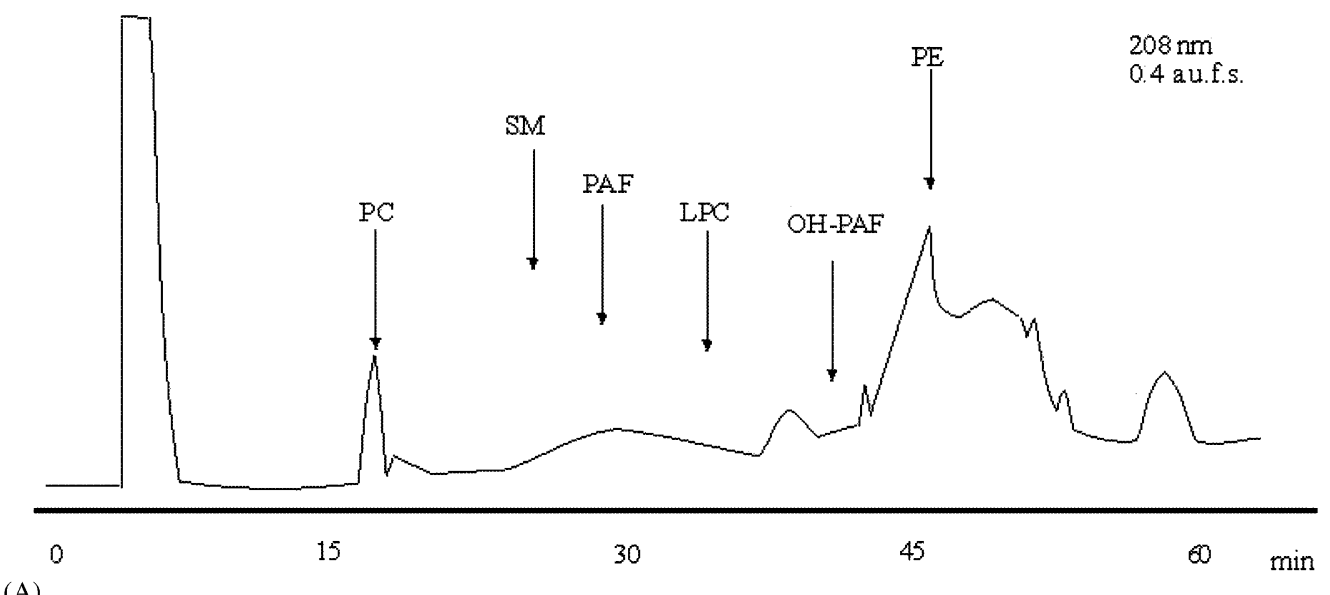

(A)

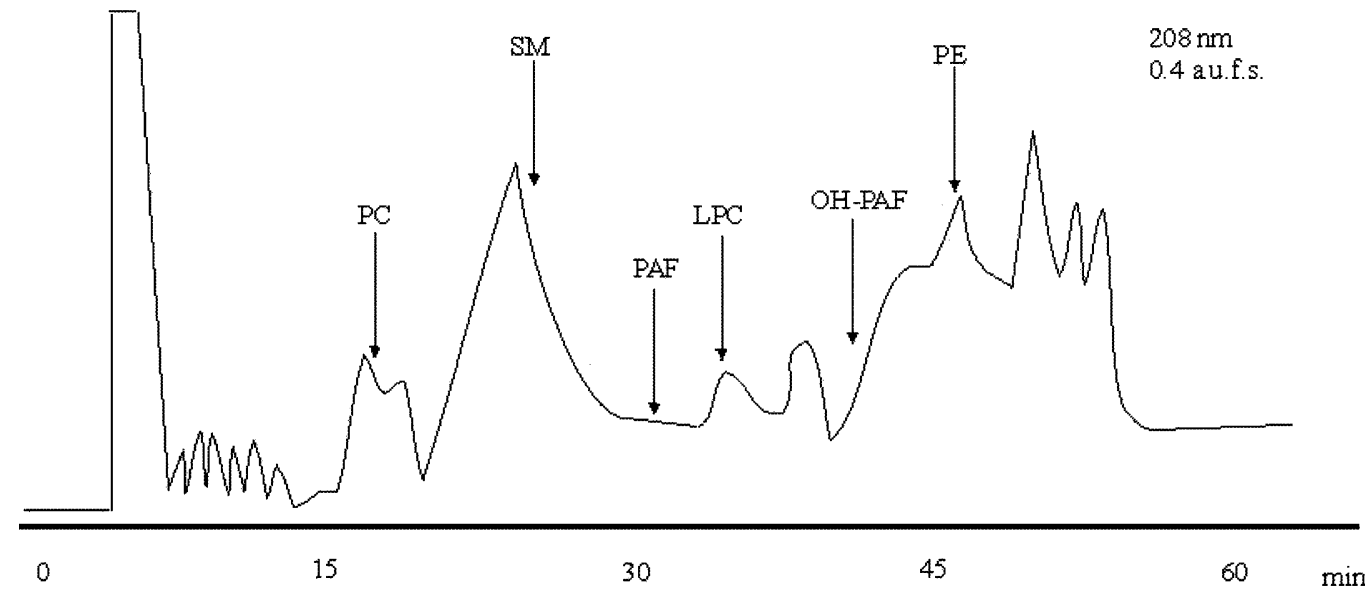

(B)

FIG. 1. (A) Representative HPLC separation of polar lipids from periodontal patients blood on a normal-phase $\mathrm{NH}_{2}$ column. The solvent system consisted of an isocratic elution with 100\% solvent A (acetonitrile/methanol, 70:30) for 35 min followed by a linear gradient to $100 \%$ solvent B (methanol) for $5 \mathrm{~min}$, a hold for $5 \mathrm{~min}$ in $100 \%$ solvent B followed by a linear gradient to $100 \%$ solvent $C$ (water) for $5 \mathrm{~min}$, and finally a hold in $100 \%$ solvent $C$ for $10 \mathrm{~min}$. The eluted substances were detected using ultraviolet detection at $208 \mathrm{~nm}$. (B) Representative HPLC separation of polar lipids from healthy volunteers blood. HPLC conditions were the same as already described. PE, phosphatidylethanolamine; PC, phosphatidylcholine; SM, sphingomyelin; LPC, lysophosphatidylcholine; OH-PAF, hydroxyl-PAF; a.u.f.s., absorption units full scale. 
$\mathrm{mM}, \mathrm{pH} 7.4$ ), human serum acetylhydrolase and the examined sample in BSA at $2.5 \mathrm{mg} / \mathrm{ml}$ of saline were added to a pre-warmed $\left(37^{\circ} \mathrm{C}\right)$ test tube. The enzymatic system was incubated at $37^{\circ} \mathrm{C}$ and, at different time intervals, aliquots were taken to test their ability to induce washed rabbit platelet aggregation.

\section{Mild alkaline hydrolysis and re-acetylation}

An amount of the examined samples was subjected to mild alkaline hydrolysis and re-acetylation, while another amount of these lipids was only subjected to acetylation. The biological activities of the lipids derived from the aforementioned chemical reactions were then tested on washed rabbit platelets. These procedures were carried out according to Demopoulos et al. ${ }^{6}$

\section{Statistical analysis}

Data are expressed as mean values \pm standard deviations. For the statistical comparison of values the $t$ test was used.

\section{Results and discussion}

The HPLC purification step from the PAF isolation procedure $^{24}$ has been modified in the present study, in order to achieve separation of hydroxyl-PAF analogue from PAF itself. The purified and identified hydroxyl-PAF analogue from $\mathrm{GCF}^{7}$ was used as standard and it was eluted under the HPLC conditions described in Methods. Subsequently, fractions of $1 \mathrm{ml}$ were manually collected and tested for their ability to induce washed rabbit platelet aggregation. The same procedure was performed for semi-synthetic PAF. The results showed that PAF and the hydroxyl-PAF analogue were completely separated under the aforementioned HPLC conditions with retention times of 30-34 min and 38-42 min, respectively.

Blood samples from patients and from periodontally healthy volunteers were separately purified under the same HPLC conditions, and fractions of 1 $\mathrm{ml}$ were manually collected from 25 to $48 \mathrm{~min}$. Representative chromatographs along with the elution times of standard lipids are shown in Fig. 1A,B. The PAF and the PAF-like activity were estimated in each HPLC fraction by bioassay studies based on washed rabbit platelets. PAF derived from patients' blood and the one from healthy volunteers was detected from 30 to $33 \mathrm{~min}$ and from 29 to $32 \mathrm{~min}$, respectively, while hydroxyl-PAF analogue was detected from 37 to $39 \mathrm{~min}$ and from 36 to $39 \mathrm{~min}$, respectively (Fig. 1). Fractions from 37 to 39 min were pooled together, representing hydroxyl-PAF analogue from periodontal patients. The same procedure was followed for fractions from 36 to $39 \mathrm{~min}$ representing hydroxyl-PAF analogue from healthy volunteers.

These hydroxyl-PAF analogues desensitized platelets against PAF and themselves, but not against thrombin (Fig. 2). Furthermore, the aggregation induced by these molecules was inhibited by BN 52021, a specific PAF-receptor antagonist. As we have previously reported, ${ }^{7}$ the concentration of the hydroxyl-PAF analogue, which induces 50\% of the maximum aggregation $\left(\mathrm{EC}_{50}\right)$, based on phosphorus determination, was in the order of $0.1 \mu \mathrm{M}$ (final concentration), while its threshold concentration allowing platelet aggregation was in the order of $0.03 \mu \mathrm{M}$ (final concentration), being less active than $\mathrm{PAF}$, which induces aggregation with a $\mathrm{EC}_{50}$ value in the order of $0.1 \mathrm{nM}$ (final concentration). Incubation of the hydroxyl-PAF analogues with human serum acetylhydrolase resulted in their time-dependent inactivation, with a rate identical to that of PAF, suggesting the presence of an acetyl group at the sn2 position of a glyceryl backbone. Mild alkaline hydrolysis rendered the hydroxyl-PAF analogues

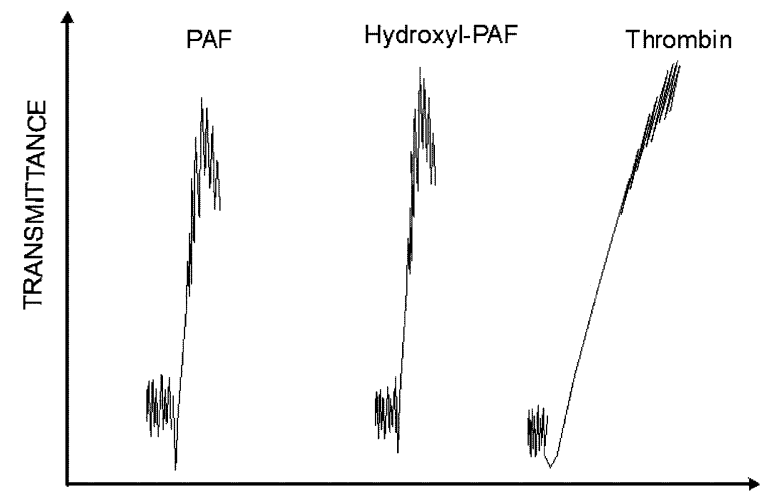

(A)

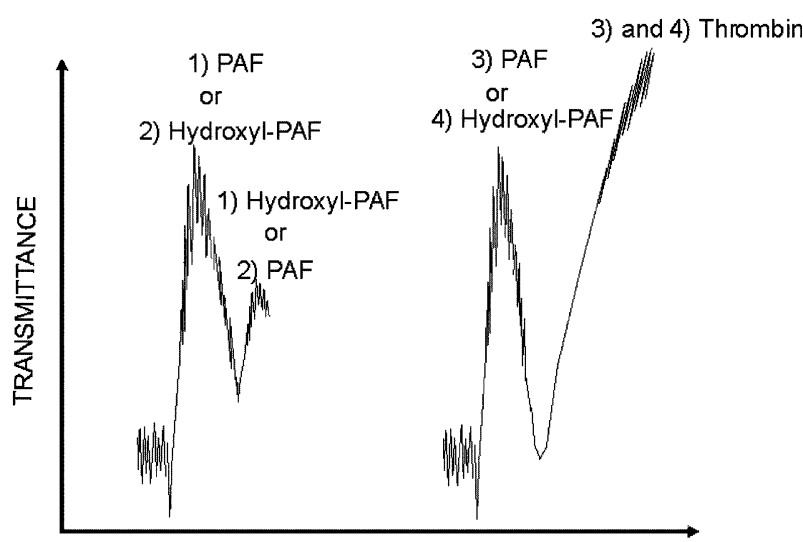

(B)

FIG. 2. (A) Reversible washed rabbit platelet aggregation curves induced by semi-synthetic PAF and by the hydroxyl$\mathrm{PAF}$ analogue isolated from blood, and the irreversible washed rabbit platelet aggregation curve induced by thrombin. (B) Desensitization experiments with washed rabbit platelets: (1) PAF against hydroxyl-PAF analogue; (2) hydroxyl-PAF analogue against PAF; (3) PAF against thrombin; (4) hydroxyl-PAF analogue against thrombin. 


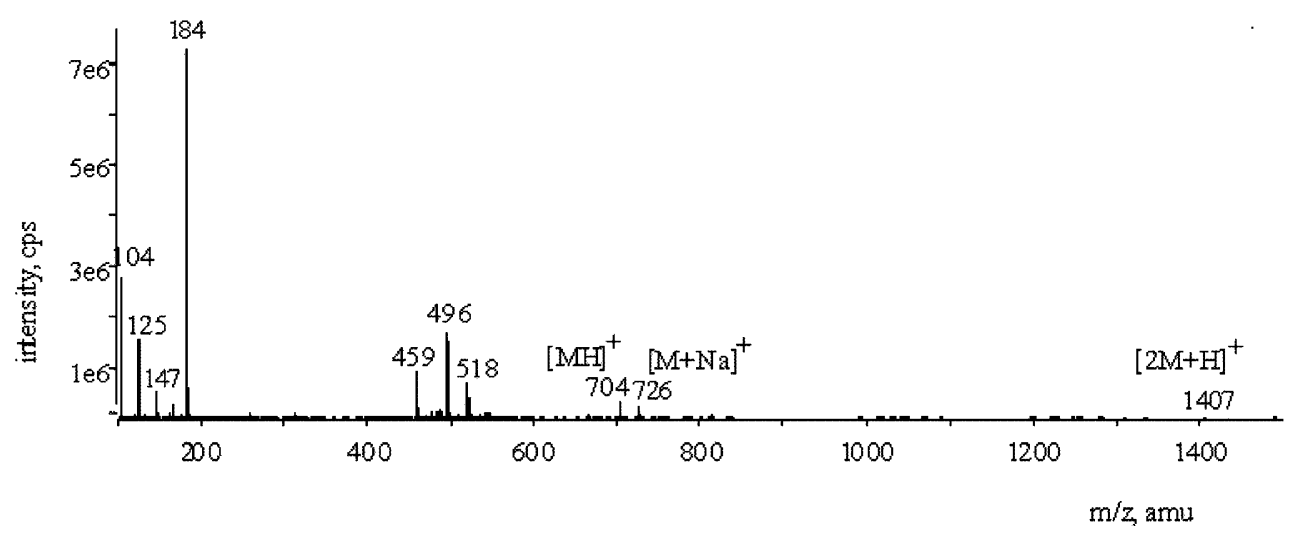

FIG. 3. Positive ion electrospray mass spectrum of the HPLC fraction of the hydroxyl-PAF analogue isolated from blood.

completely inactive while re-acetylation of the chloroform-soluble fractions, resulting from the earlier hydrolysis, increased their biological activity. Acetylation of the initial hydroxyl-PAF analogues resulted in an increase of their biological activity, identical to that observed after the re-acetylation of the chloroform-soluble fractions.

Even though the retention times of the hydroxylPAF analogues as well as their biological characteristics were almost identical to that of the hydroxylPAF analogue from $\mathrm{GCF}^{7}$ these lipids isolated from blood samples were consequently subjected to ESI analysis, in order to confirm that all these inflammatory molecules share the same structure.

The positive ESI spectra of the fractions were identical (Fig. 3) and showed $\left[\mathrm{M}^{+} \mathrm{H}\right]^{+},\left[\mathrm{M}^{+} \mathrm{Na}\right]^{+}$ and $\left[2 \mathrm{M}^{+} \mathrm{H}\right]^{+}$ions, at mass-to-charge ratio $(\mathrm{m} / z)$ 704, 726 and 1407, respectively. The ions at $m / z$ 184, 147, 125 and 104, which correspond to fragments of the phosphocholine moiety, were also present.

The ESI-MS/MS spectrum of the fragment ion at $\mathrm{m} /$ $z 704$ is represented in Fig. 4. The abundant ion at $\mathrm{m} /$ $z 184$ is corresponded to the phosphocholine moiety. The positive fragment ion at $\mathrm{m} / z 686$ may correspond to the loss of $\mathrm{H}_{2} \mathrm{O}$, while the other fragments at $\mathrm{m} / z$ 645 and 521 should correspond to the loss of $\left[\mathrm{N}\left(\mathrm{CH}_{3}\right)_{3}\right]$ and of the polar choline phosphate head group as neutral fragment $183 \mathrm{Da}$ from the molecule. Identical fragmentations were observed at the ESIMS/MS spectrum of the positive ion at $m / z 726$ (Fig. 5), with fragment ions at $m / z 667\left[\mathrm{M}^{+} \mathrm{Na}-\mathrm{N}\left(\mathrm{CH}_{3}\right)_{3}\right]^{+}$, at $m / z 542\left[\mathrm{M}^{+} \mathrm{Na}-183\right]^{+}$and at $m / z$ 184. These

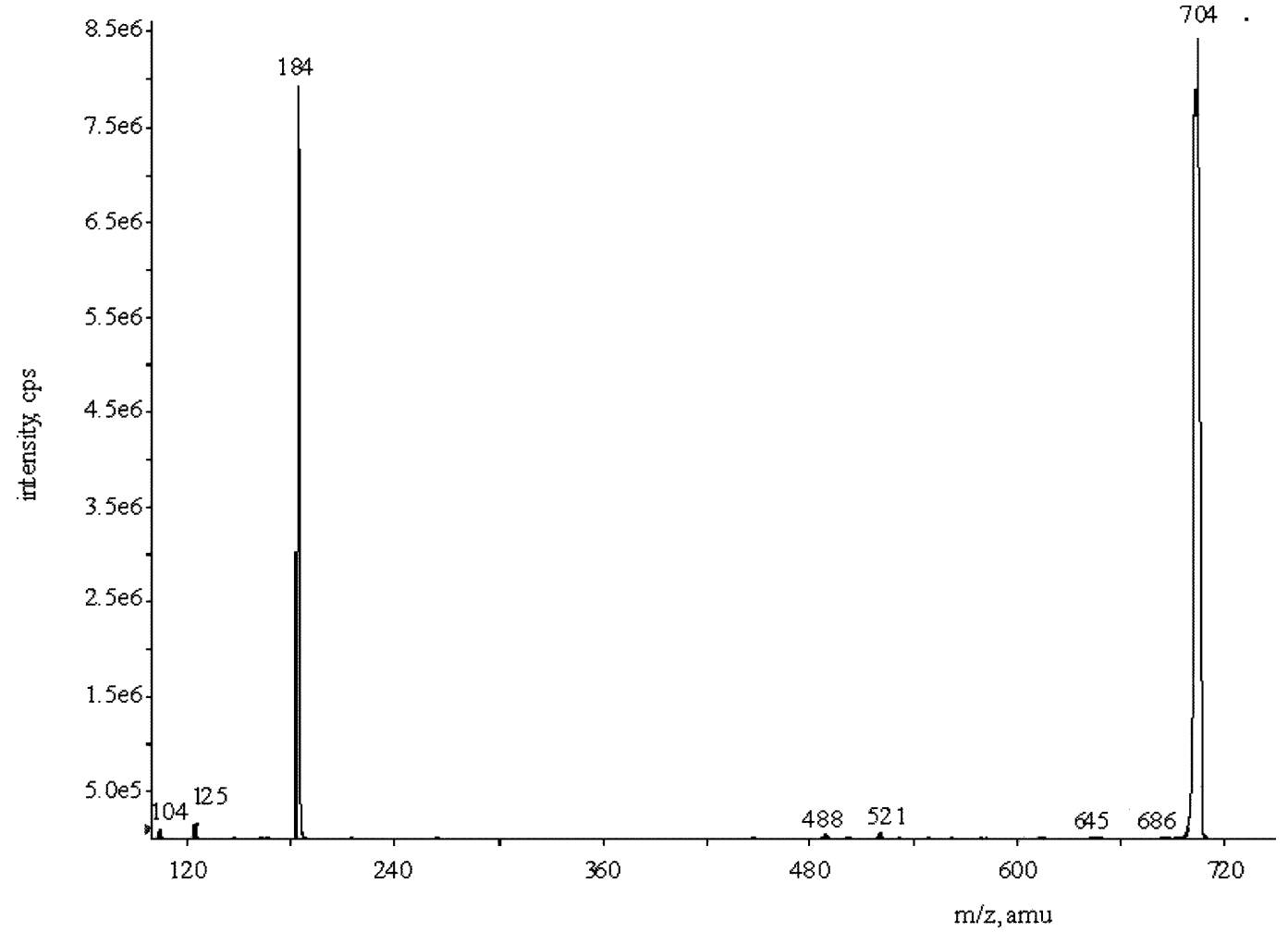

FIG. 4. Positive ion electrospray MS/MS spectrum of the ion at $m / z 704$. 


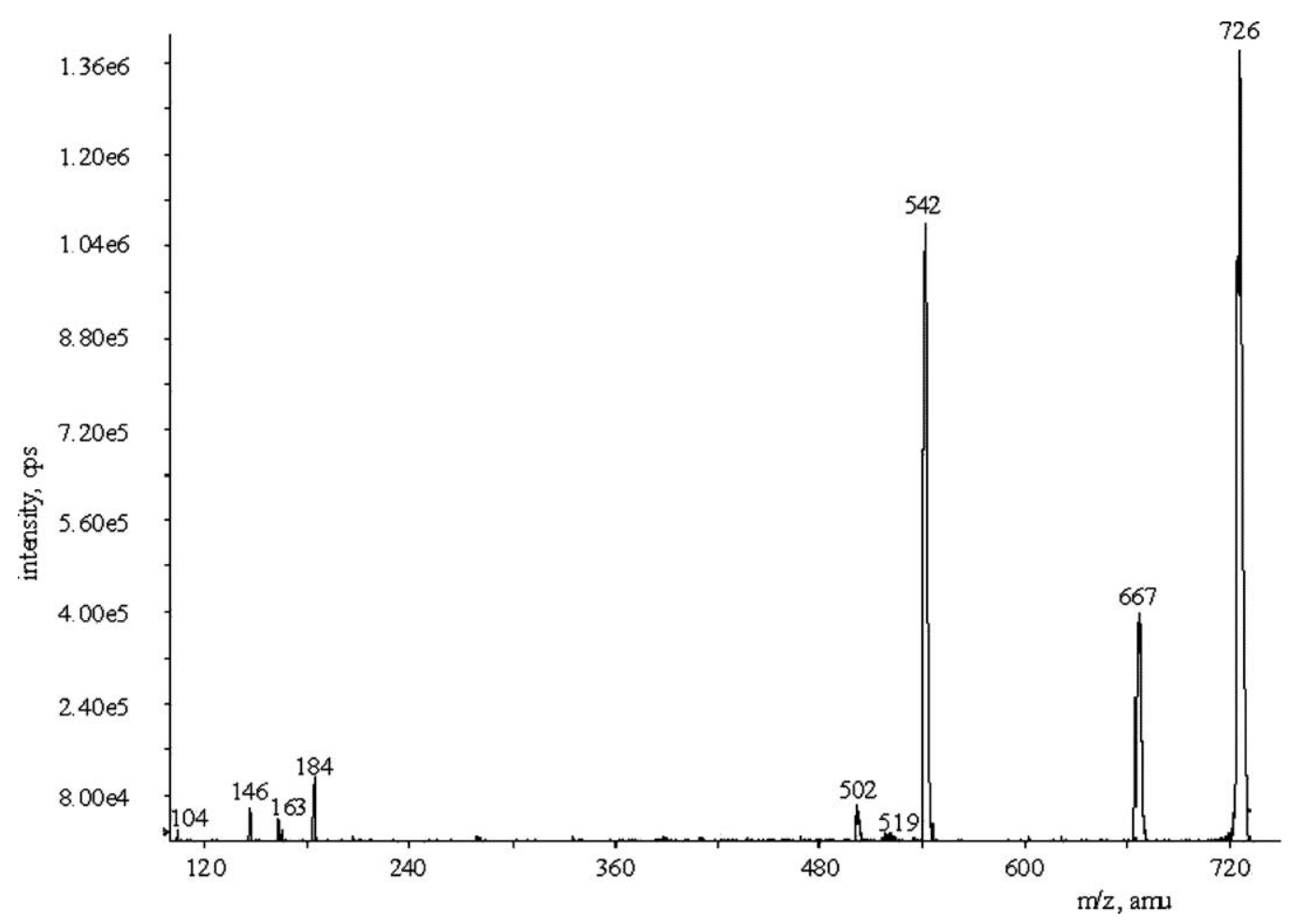

FIG. 5. Positive ion electrospray MS/MS spectrum of the ion at $m / z 726$.

fragmentations have been previously reported for sodiated phosphatidylcholine molecules [26] and for lysoglycerophosphocholine lipids [27]. In addition a positive ion at $\mathrm{m} / \mathrm{z} 502$ was present that should correspond to the loss of the acetyl group $\left[\mathrm{M}^{+} \mathrm{Na}-\right.$ $183-42]^{+}$from the $s n-2$ position of the glycerylbackbone.

The ESI-MS/MS positive spectrum of the fragment ion at $\mathrm{m} / z 1407$ (Fig. 6) confirmed that this ion is corresponded to $\left[2 \mathrm{M}^{+} \mathrm{H}\right]^{+}$.

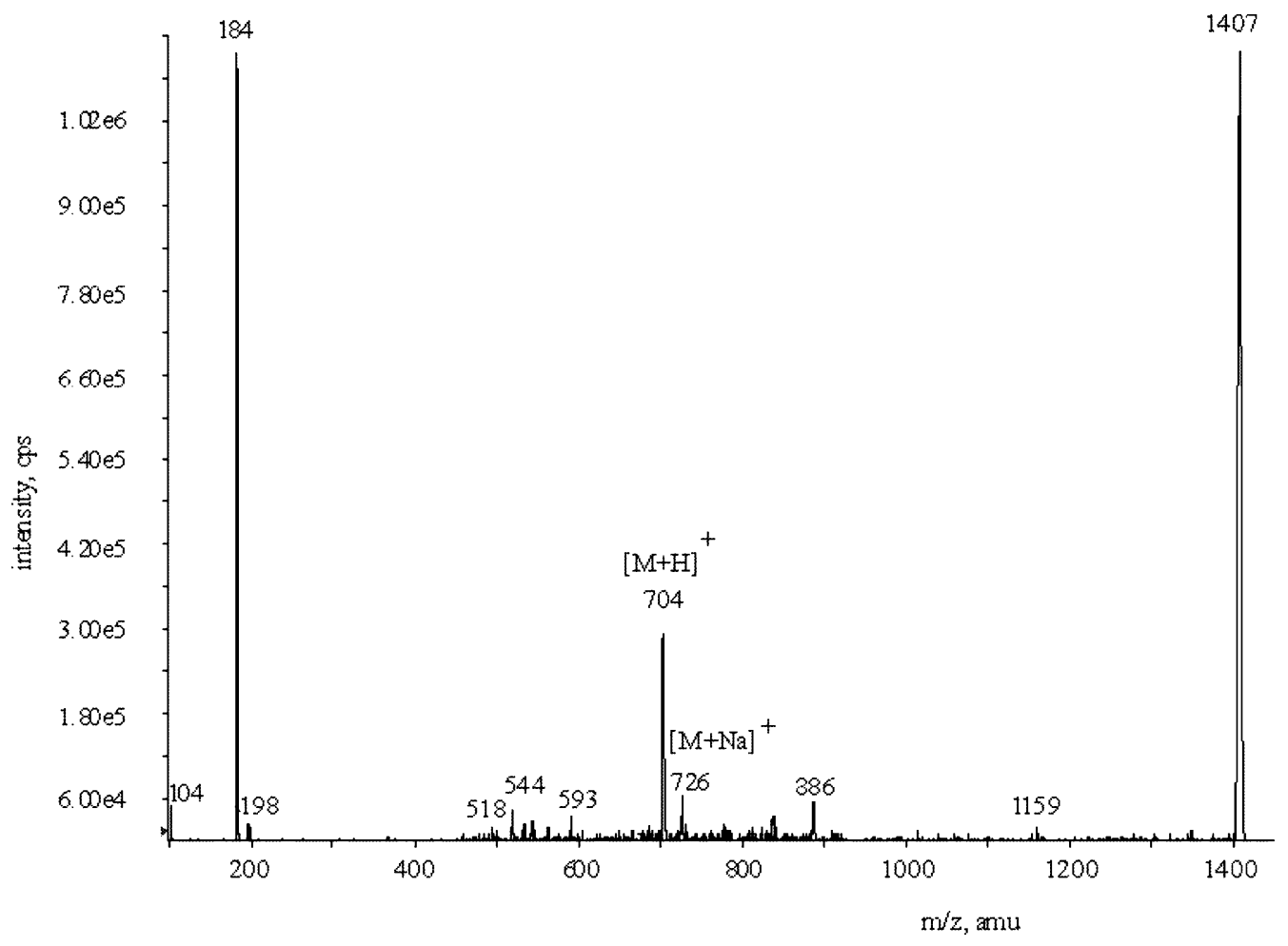

FIG. 6. Positive ion electrospray MS/MS spectrum of the ion at $m / z 1407$. 
Table 1. PAF and hydroxyl-PAF analogue levels in blood collected from five periodontally healthy volunteers as well as from five patients with chronic adult periodontitis

\begin{tabular}{lccc}
\hline & Healthy individuals & Patients & $p$ values \\
\hline PAF levels (moles/ml of blood) & $3.43 \pm 0.86 \times 10^{-14}$ & $2.47 \pm 0.93 \times 10^{-14}$ & $>0.05$ \\
Hydroxyl-PAF levels (moles/ml of blood) & $0.81 \pm 0.18 \times 10^{-14}$ & $0.74 \pm 0.29 \times 10^{-14}$ & $>0.05$ \\
Hydroxyl-PAF levels to PAF levels & $23.66 \pm 1.49$ & $30.0 \pm 1.80$ & $<0.05$ \\
\hline
\end{tabular}

All the presented findings show that the biological active lipid, present in periodontal patients blood as well as in healthy individuals blood, is indeed a hydroxyl-PAF analogue identical to that isolated from GCF. $^{7}$

The quantitative determination of PAF and hydroxyl-PAF analogue levels, based on PAF-like activity, showed that in patients with periodontitis PAF levels as well as hydroxyl-PAF analogue levels were not different from those of healthy individuals (Table 1). A statistically significant increase was detected in the ratio of hydroxyl-PAF analogue levels to PAF levels in periodontal patients, suggesting that this bioactive lipid may play a role in oral inflammation.

In conclusion, the existence of an endogenous hydroxyl-PAF analogue in human blood is reported for the first time. This biologically active lipid is identical to that isolated from GCF samples. Although the precise source as well as the possible pathophysiological role of hydroxyl-PAF analogue remains to be established, it seems that the infected periodontium can be a source of potentially inflammatory mediators. On the contrary, since PAF and PAF-like oxidized phospholipids are implicated in atherosclerosis and cardiovascular diseases; the hydroxylPAF analogue could be a link between periodontal disease and atherosclerosis. However, much remains to be understood about the exact mechanism through which PAF-like molecules exert their action.

\section{References}

1. Socransky SS, Haffajee AD. Evidence of bacterial etiology: a historical perspective. Periodontol 1994; 2000: 7-25.

2. Beck J, Garcia R, Heiss G, Vokonas PS, Offenbacher S. Periodontal disease and cardiovascular disease. J Periodontol 1996; 67: 1123-1137.

3. Page RC. The role of inflammatory mediators in the pathogenesis of periodontal disease. J Periodont Res 1991; 26: 230-242.

4. Emingil G, Cinarcik S, Baylas $H$, Huseyinov A. Levels of plateletactivating factor in gingival crevicular fluid and gingival tissue in specific periodontal diseases. J Periodontol 2001; 72: 1032-1037.

5. Baltas G, Kotsifaki H, Antonopoulou S, Kipioti A, Demopoulos CA. Implication of PAF and acetylhydrolase (PAF-AH) activity in periodontal disease. Adv Exp Med Biol 1996; 416: 135-141.

6. Demopoulos CA, Pinckard RN, Hanahan DJ. Platelet-activating factor. Evidence for 1-O-alkyl-2-acetyl-sn-glyceryl-3-phosphoryl-choline as the active component. (A new class of lipid chemical mediators.). J Biol Chem 1979; 254: 9355-9358.

7. Antonopoulou S, Demopoulos CA, Argyropoulos D, Baltas G, Kotsifaki $\mathrm{H}$, Diamanti-Kipioti A. Identification of a new endogenous platelet- activating factor-like molecule in gingival crevicular fluid. Biochem $J$ 1998; 330: $791-794$.

8. McManus LM, Pinckard RN. PAF, a putative mediator of oral inflammation. Crit Rev Oral Biol Med 2000; 11: 240-258.

9. McManus LM, Marze BT, Schiess AV. Deficiency of salivary PAF in edentulous individuals. J Periodontal Res 1990; 25: 347-351.

10. Ribaldi E, Guerra M, Mezzasoma AM, Staffolani N, Goracci G, Gresele P. PAF levels in saliva are regulated by inflammatory cells. $J$ Periodontal Res 1998; 33: 237-241

11. Armitage GC. Periodontal infections and cardiovascular disease-how strong is the association? Oral Dis 2000; 6: 335-350.

12. Kolltveit KM, Eriksen HM. Is the observed association between periodontitis and atherosclerosis cause? Eur J Oral Sci 2001; 109: 2-7.

13. Beck JD, Pankow J, Tyroler HA, Offenbacher S. Dental infections and atherosclerosis. Am Heart J 1999; 138: S528-S533.

14. Kinane DF. Periodontal diseases contributions to cardiovascular disease: an overview of potential mechanism. Ann Periodontol 1998; 3: 142150 .

15. Triggiani M, Schleimer RP, Warner JA, Chilton FH. Differential synthesis of 1-acyl-2-acetyl-sn-glycero-3-phosphocholine and platelet-activating factor by human inflammatory cells. J Immunol 1991; 146: 660-666.

16. Tokumura A, Sumida T, Toujima M, Kogure K, Fukuzawa K. Plateletactivating factor (PAF)-like oxidized phospholipids: relevance to atherosclerosis. Biofactors 2000; 13: 29-33

17. Brocheriou I, Stengel D, Mattsson-Hulten L, et al . Expression of plateletactivating factor receptor in human cardiovascular atherosclerotic plaques: relevance to progression of atherosclerosis. Circulation 2000; 21: $2569-2575$

18. Montrucchio G, Alloatti G, Camussi G. Role of platelet-activating factor in cardiovascular pathophysiology. Phys Rev 2000; 80: 1669-1699.

19. Liapikos TA, Antonopoulou S, Karabina SA, Tsoukatos DC, Demopoulos $\mathrm{CA}$, Tselepis AD. Platelet-activating factor formation during oxidative modification of low-density lipoprotein when PAF-acetylhydrolase has been inactivated. Biochim Biophys Acta 1994; 1212: 353-360.

20. Karantonis HC, Antonopoulou S, Demopoulos CA. A biochemical approach of the antiatherogenic properties of olive oil and its protective role against coronary heart disease, Proceedings of the 93rd AOCS Annual Meeting \& Expo, Montreal, Quebec, 5-8 May.

21. Stafforini DM, McIntyne TM, Carter ME, Prescott SM. Human plasma platelet-activating factor acetylhydrolase. J Biol Chem 1987; 262: 42234230

22. Demopoulos CA, Andrikopoulos NK, Antonopoulou S. A simple and precise method for the routine determination of platelet-activating factor in blood and urine. Lipids 1994; 29: 305-309.

23. Bligh EG, Dyer WJ. A rapid method of total lipid extraction and purification. Can J Biochem Physiol 1959; 37: 911-917.

24. Nomikos T, Karantonis HC, Fragopoulou E, Demopoulos CA. One step separation system for the main phospholipids, glycolipids and phenolics by normal phase HPLC. Application to polar lipids extracts from olive and sunflower oils. J Liquid Chromatogr Rel Technol 2002; 25: 137-149.

25. Lazanas M, Demopoulos CA, Tournis S, Koussissis S, Labrakis-Lazanas K, Tsarouhas X. PAF of biological fluids in disease: IV. Levels in blood in allergic reactions induced by drugs. Arch Dermatol Res 1988; 280: 124126.

26. Brugger B, Erben G, Sandhoff R, Wieland FT, Lehmann WD. Quantitative analysis of biological membrane lipids at the low picomole level by nano-electrospray ionization tandem mass spectrometry. Proc Natl Acad Sci USA 1997; 94: 2339-2344.

27. Khaselev N, Murphy RC. Electrospray ionization mass spectrometry of lysoglycerophosphocholine lipid subclasses. J Am Soc Mass Spectrom 2000; 11: 283-291.

\section{Received 1 April 2003 \\ Accepted 16 June 2003}




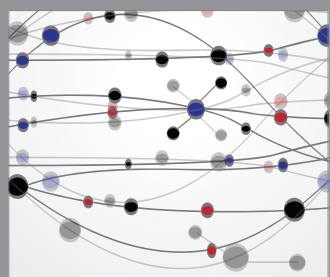

The Scientific World Journal
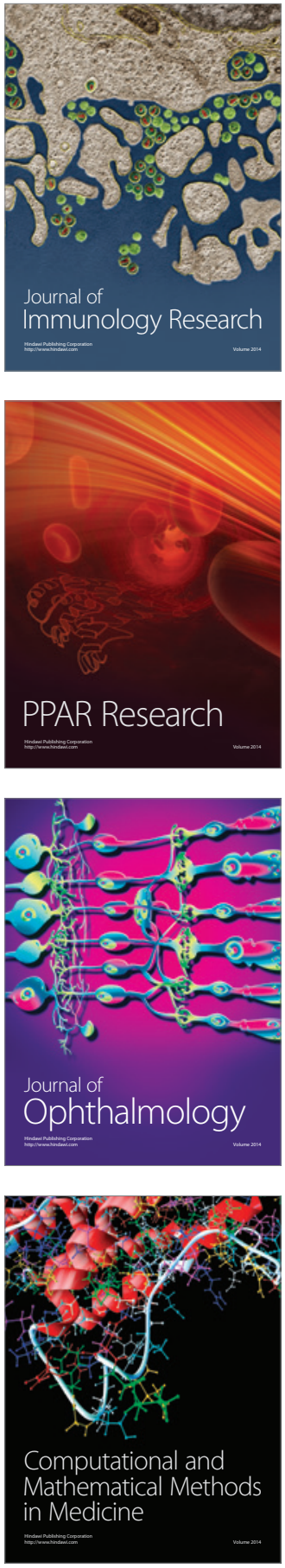

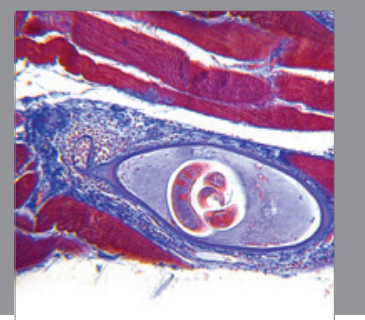

Gastroenterology

Research and Practice
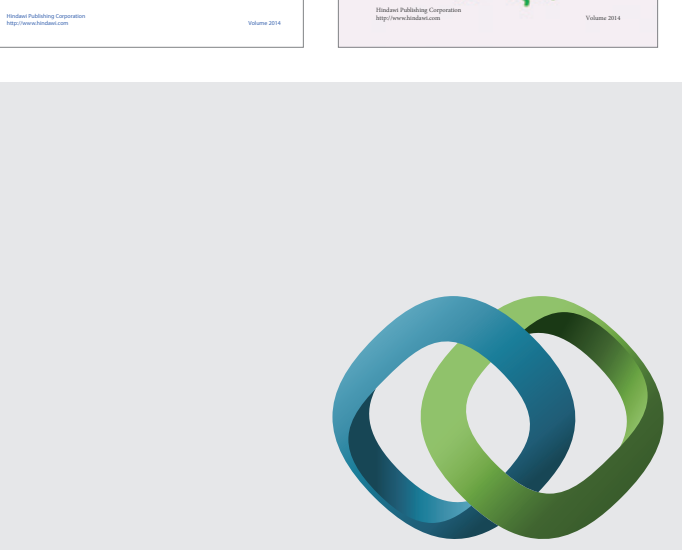

\section{Hindawi}

Submit your manuscripts at

http://www.hindawi.com
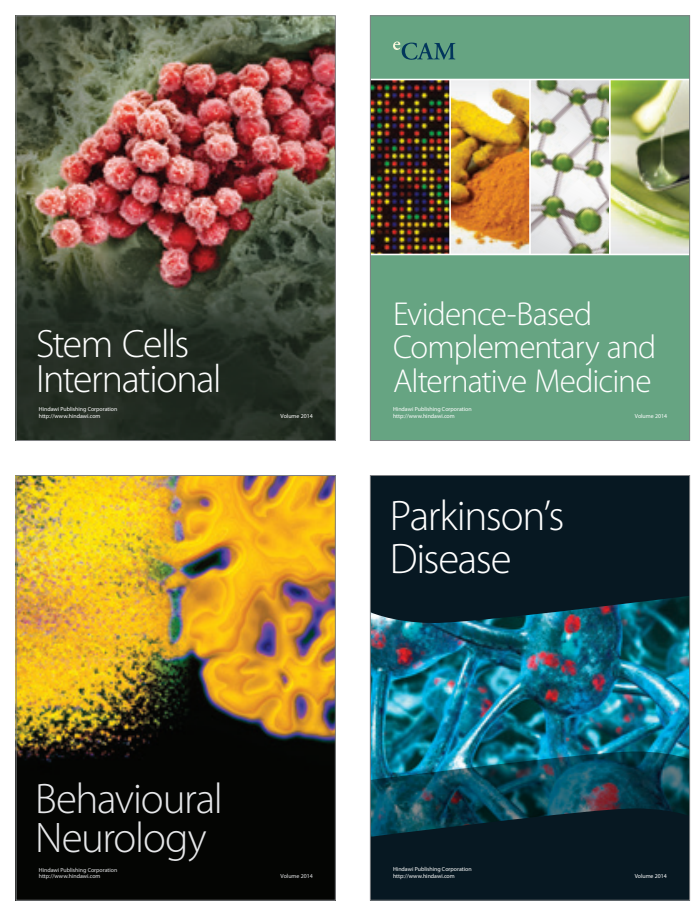

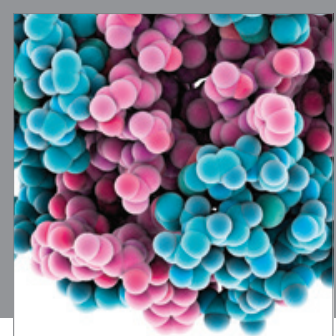

Journal of
Diabetes Research

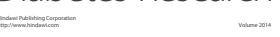

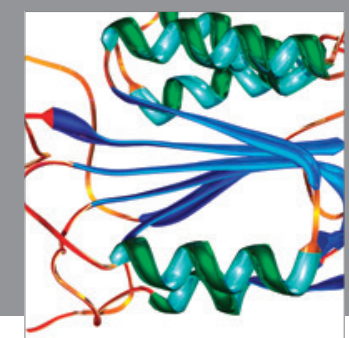

Disease Markers
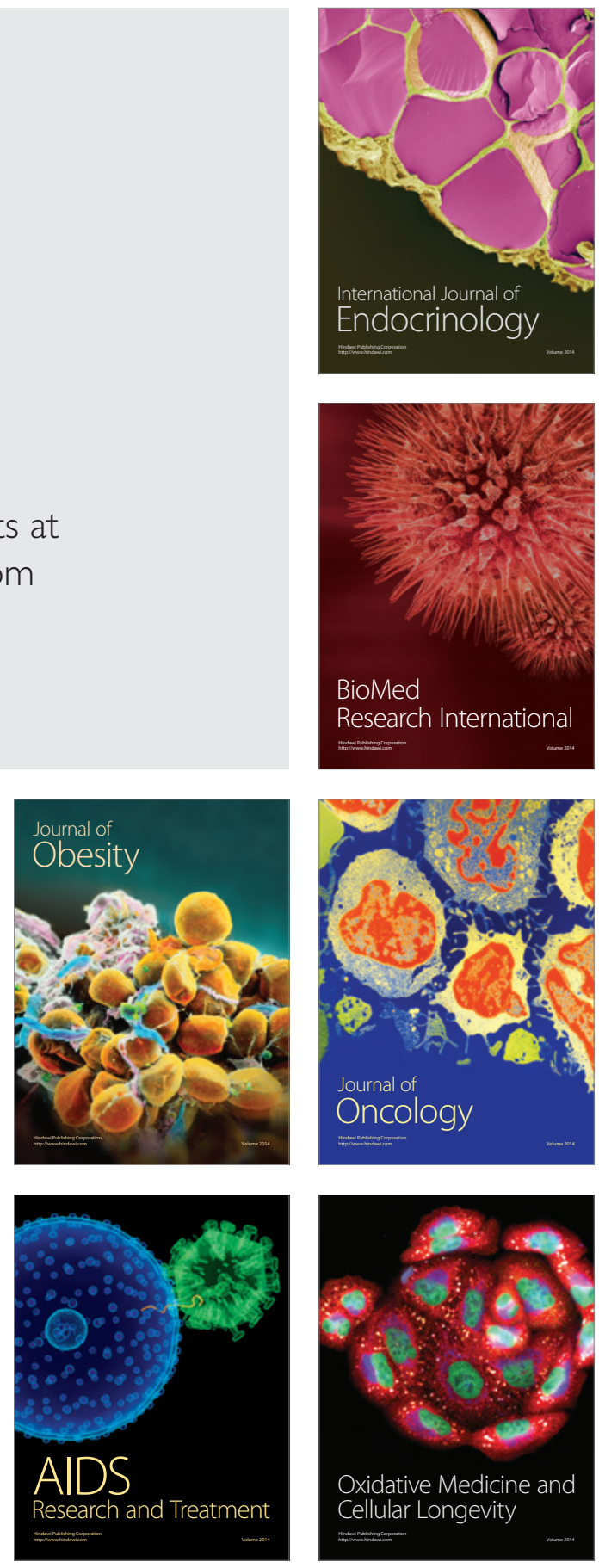